\title{
Electronic structure near an antiphase boundary in magnetite
}

\author{
R. Arras, L. Calmels, and B. Warot-Fonrose \\ CEMES-CNRS, Université de Toulouse, 29 rue Jeanne Marvig, BP 94347, F-31055 Toulouse Cedex 4, France \\ (Received 4 December 2009; revised manuscript received 24 February 2010; published 26 March 2010)
}

\begin{abstract}
We present a first-principles calculation of the electronic structure near an antiphase boundary (APB) in magnetite, with the shift vector along the [110] direction and a $\{110\}$ boundary plane. We have first used the local spin-density approximation to calculate the ground-state energy of a supercell with two magnetite grains separated by an APB, and found that this energy is lower when magnetite grains are coupled ferromagnetically. The LDA $+U$ approximation has later been used for an accurate description of the density of states near the boundary. Results show that localized electron states can exist in the vicinity of the structural defect. We give a clear description of the dispersion of these APB states.
\end{abstract}

DOI: 10.1103/PhysRevB.81.104422 PACS number(s): 75.47.Lx, 73.20.-r, 75.10.Lp, 75.30.Kz

\section{INTRODUCTION}

During the last 20 years, an intense research activity has been focused on the discovery of new magnetic materials for spintronic devices. Magnetic oxides could particularly be interesting for this purpose: they present a wide variety of electronic structures and magnetic behaviors, and could be used either as magnetic insulating barriers in spin filters, or as magnetic electrodes in magnetic tunnel junctions (MTJs). This is, for instance, the case of magnetite $\mathrm{Fe}_{3} \mathrm{O}_{4}$, which is ferrimagnetic and half metallic above the Verwey temperature $\left(T_{V}=120 \mathrm{~K}\right),{ }^{1}$ and becomes an insulator below $T_{V}{ }^{2,3}$ The half-metallic behavior of magnetite is preserved up to the high Curie temperature $T_{C}=858 \mathrm{~K}$. MTJs using magnetite electrodes should consequently possess exceptional magnetoresistive effects at temperatures well above room temperature.

Transport and magnetic properties of spintronic devices using magnetite layers are unfortunatly strongly affected by the structural defects of this oxide. The most common structural defects, which appear in spinel ferrites such as magnetite, are the so-called antiphase boundaries (APBs). ${ }^{4,5}$ APBs are two-dimensional structural defects in which atoms located on both sides of the boundary are linked by a shift vector which differs from a lattice vector. These defects exist in bulk samples. They also arise during the growth of thin magnetite layers on a crystal substrate. The characteristics of the APBs depend in this case on the nature of the substrate and on the growth conditions.

APBs can exist with seven different shift vectors in magnetite. ${ }^{6,7}$ They have been observed by transmission electron microscopy and by scanning tunneling microscopy. ${ }^{8}$ Eerenstein et al. have measured the proportion of APBs corresponding to each of the seven different shift vectors and determined the orientation of the APBs as a function of their shift vector. ${ }^{9,10}$ When magnetite is grown on a $\mathrm{MgO}(001)$ substrate, the shift vectors $(1 / 4) a_{0}[110],(1 / 4) a_{0}[1 \overline{1} 0]$, and $(1 / 2) a_{0}[100]$ have been observed, where $a_{0}$ is the lattice parameter of magnetite. These shift vectors are all contained in the plane of the magnetite films. The first two of these vectors (and the corresponding APBs) are equivalent and they are perpendicular to the APB layer. The three shift vectors mentioned above almost correspond to $44.5 \%$ of the observed APBs. The four shift vectors $(1 / 4) a_{0}[011]$, $(1 / 4) a_{0}[0 \overline{1} 1],(1 / 4) a_{0}[101]$, and $(1 / 4) a_{0}[\overline{1} 01]$ have also been observed. Each of these four vectors, which are not parallel to the $\mathrm{Fe}_{3} \mathrm{O}_{4}$ film, have been observed with a proportion which varies between $12.5 \%$ and $15.5 \%$. These vectors are mainly associated to APBs in a $\{100\}$ or a $\{310\}$ plane. The density of APBs can vary with parameters like the growth temperature or the oxygen flux during growth. It generally increases when the film thickness decreases (the size of a domain delimited by APBs increases as the square root of the film thickness). Magen et al. ${ }^{11}$ have shown that the density of APBs can be lowered in magnetite by inserting a very thin $\mathrm{Fe}$ or $\mathrm{Cr}$ layer between the $\mathrm{MgO}$ substrate and the magnetite film.

The consequences of APBs on magnetic and transport properties have been studied in details during the recent years. The global magnetic coupling between two magnetite domains separated by an APB can be either ferromagnetic or antiferromagnetic, depending on the direction and on the shift vector of the APB. Goodenough ${ }^{12}$ and Kanamori ${ }^{13}$ have proposed general laws which explain the microscopic origin of this coupling: these authors have described the magnetic coupling as a function of the distance between atoms ( $\mathrm{Fe}$ and oxygen in our case), and as a function of the angle between chemical bonds. These laws can, in principle, be used to predict the nature of the magnetic coupling for a given APB. In practice, however, such predictions may be complicated because several chemical bonds, which favor opposite magnetic coupling, must be considered for a given APB. According to the laws of Goodenough and Kanamori, Celotto and Eerenstein have suggested that magnetite grains are ferromagnetically coupled across an APB, when the APB is in a $\{100\}$ plane. ${ }^{9,10}$ The coupling becomes antiferromagnetic when the APB is in a $\{310\}$ plane. Ferromagnetic or antiferromagnetic coupling can both exist for $\{110\}(1 / 4) a_{0}[110]$ APBs, depending on the influence of all the APBs which are delimitating the magnetite grains under consideration. Margulies used the same laws to study the same APBs, but obtained different conclusions than that of Eerenstein, on the magnetic coupling across these APBs. ${ }^{7}$ The magnetic coupling between grains across an APB has a strong influence on the macroscopic magnetic behavior of magnetite samples. It is, for instance, more difficult to saturate an epitaxial film 
than a bulk magnetite crystal, ${ }^{7}$ and the saturation magnetization has been found lower for ultrathin films than for bulk samples. ${ }^{14,15}$ The reason for this difference has been attributed to the larger number of APBs in thin films. ${ }^{7}$ Finally, epitaxial $\mathrm{Fe}_{3} \mathrm{O}_{4}$ films can exhibit a superparamagnetic behavior for thicknesses below $5 \mathrm{~nm}$, where the size of the domains becomes very small. ${ }^{16,17}$ Antiphase boundaries also have a great influence on the transport properties of magnetite samples: the electric resistance, which is expected to be high across a single APB with antiferromagnetic coupling, increases strongly when many APBs are present in the sample. $^{6}$

First-principles calculations based on the densityfunctional theory have been performed to calculate the electronic structure of bulk $\mathrm{Fe}_{3} \mathrm{O}_{4}$. The local spin-density approximation (LSDA) and the generalized gradient approximation have been used to study the high-temperature cubic phase of magnetite, ${ }^{18-22}$ confirming the half metallicity of this oxide. The first-order metal-insulator Verwey transition which occurs at $T_{V}$ has been studied with more elaborated methods like the self-interaction corrected local spindensity approximation ${ }^{23}$ or the LDA $+U$ method. ${ }^{24,25}$ These methods have also been used to calculate the density of states (DOS) for the high-temperature phase of magnetite with correct values for the band gaps. ${ }^{26,27}$ These calculations only concern bulk magnetite crystals. Results on the electronic structure near structural defects in magnetite cannot be found in the literature. Information on the electronic structure near antiphase boundaries would actually be very useful to understand the physical properties of true magnetite samples in which the transport and magnetic behavior can be strongly affected by APBs.

In this paper we have used the first-principles LSDA and $\mathrm{LDA}+U$ methods to calculate the electronic structure near the APB $\{110\}(1 / 4) a_{0}[110]$ in the high-temperature cubic phase of magnetite. The density of states near this structural defect is compared to values calculated for bulk magnetite. Our paper is organized as follows. In the Sec. II we will describe the computational methods and the supercells which have been used for the calculations of the electronic structure. In Sec. III, we describe the electronic structure in the vicinity of a $\{110\}(1 / 4) a_{0}[110]$ APB. We finally conclude in Sec. IV.

\section{DESCRIPTION OF THE SUPERCELL AND COMPUTATIONAL DETAILS}

The simplest supercell which we have used for the calculation of the electronic structure in the vicinity of the APB $\{110\}(1 / 4) a_{0}[110]$ contains ten (110) atomic layers and is represented in Fig. 1. The first four and the last four atomic layers of the supercell shown in Fig. 1 are organized with the same stacking as in the high-temperature phase of bulk magnetite (inverse spinel structure with space group $F d-3 m$ and lattice parameter $a_{0}=0.8397 \mathrm{~nm}$ ). These layers correspond to two periods of $\mathrm{Fe}_{3} \mathrm{O}_{4}$ in the [110] direction, and form a face-centered cubic lattice of oxygen atoms with iron atoms located in $1 / 8$ of the tetrahedral and half of the octahedral atomic sites (respectively, $\mathrm{Fe}_{A}$ and $\mathrm{Fe}_{B}$ atoms) according to

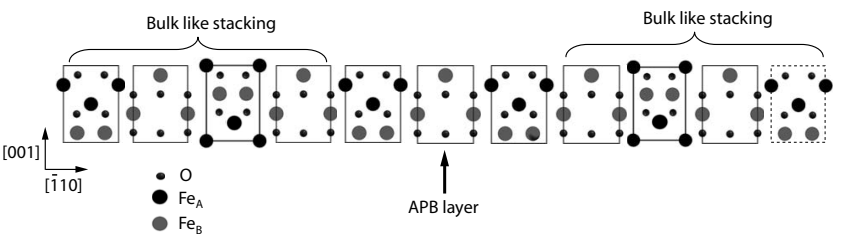

FIG. 1. Atomic structure of the supercell with a size of $1.48 \mathrm{~nm}$ in the [110] direction. The stacking of the atomic layers along [110] is represented from the left to the right of the figure. The $\{110\}(1 / 4) a_{0}[110]$ APB is indicated by an arrow. The last atomic layer drawn with a dashed line on the right-hand side of the figure is the periodic image of the first atomic layer on the left-hand side.

the formula $\left[\mathrm{Fe}^{3+}\right]_{A}\left[\mathrm{Fe}^{2+} ; \mathrm{Fe}^{3+}\right]_{B} \mathrm{O}_{4}^{2-} . \mathrm{Fe}_{A}$ atoms are antiferromagnetically coupled to $\mathrm{Fe}_{B}$ atoms. This magnetic coupling is dominant in bulk magnetite. The atomic layers with bulklike stacking are followed by atomic layers which contain the APB. The oxygen-atom tetrahedra and slightly distorted octahedra surrounding iron atoms are the same as in bulk magnetite, except in the APB layer shown in Fig. 1 in which the $0.0039 \mathrm{~nm}$ buckling of oxygen atoms has been suppressed for symmetry reasons. This supercell contains 18 nonequivalent atoms and has a size of $a_{0}, \frac{a_{0}}{\sqrt{2}}$, and $\frac{5 a_{0}}{2 \sqrt{2}}$ in the directions [001], [110], and [110], respectively. The supercell is large enough to avoid artifactual interaction between APBs in adjacent cells, separated by a distance of $1.48 \mathrm{~nm}$. This supercell has been used to calculate the electronic structure of a $\{110\}(1 / 4) a_{0}[110]$ APB with ferromagnetic coupling across the APB with the LSDA and the LDA $+U$ approximations.

We have also used a supercell with 33 nonequivalent atoms and a size of $2.97 \mathrm{~nm}$ in the [110] direction. It contains two APBs separated by a distance of $1.48 \mathrm{~nm}$, and has been used to compare the electronic structure of a $\{110\}(1 / 4) a_{0}[110]$ APB with ferromagnetic coupling and antiferromagnetic coupling across the boundary. This comparison is, however, time consuming and has only been done with the LSDA approximation. For the same reasons, we have not tried to optimize the atom coordinates in the supercells by minimizing the forces, but we checked that these forces are reasonably small.

The calculations have been done with the full potential linearized augmented plane wave code WIEN $2 \mathrm{~K},{ }^{28}$ within the LSDA and LSDA $+U$ approximations. We have chosen atomic sphere radii which minimize the volume of the interstitial area between atomic spheres: the radius is of 1.9 a.u. for $\mathrm{Fe}_{A}$ atoms, 2.3 a.u. for $\mathrm{Fe}_{B}$ atoms, and 1.4 a.u. for oxygen atoms. The size of the basis which has been used to calculate the one-electron wave function depends on the parameter $R k_{\max }$ : we have used $R k_{\max }=7.0$ with the smallest supercell and $R k_{\max }=6.0$ with the biggest one. The LSDA $+U$ calculation consists in applying an orbital-dependent correction which depends on a parameter $U$. This correction enables to take into account the strong correlation between $d$ electrons and to try to cancel the self-interaction. ${ }^{29,30}$ We have used the values of $U=4.5 \mathrm{eV}$ and $J=0.56 \mathrm{eV}$ which have been proposed by Anisimov ${ }^{25}$ and Novák, ${ }^{26}$ for the $d$ orbitals of iron atoms. The irreducible wedge of the first Brillouin zone was sampled with $72 k$ vectors for all the calculations based on 


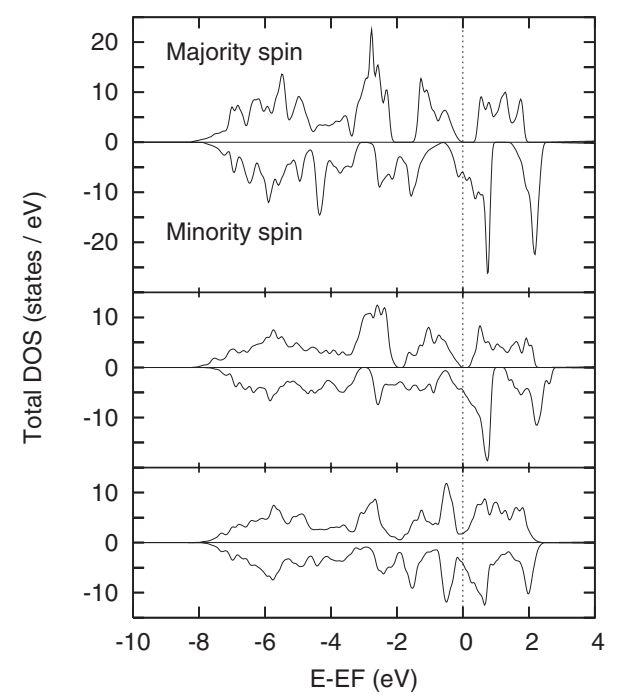

FIG. 2. Majority- and minority-spin DOS calculated with the LSDA for bulk $\mathrm{Fe}_{3} \mathrm{O}_{4}$ (upper part of the figure), for a $\{110\}(1 / 4) a_{0}[110]$ APB with ferromagnetic coupling (central part of the figure), and for a $\{110\}(1 / 4) a_{0}[110]$ APB with antiferromagnetic coupling (lower part of the figure). For the three different systems, the upper and lower curves, respectively, correspond to the DOS for majority spin and to the DOS for minority spin (multiplied by -1$)$. The DOS near the APB includes contributions from atoms in two atomic layers: the APB layer itself and one of its firstneighbor layers. The three curves describe the same number of magnetite formula units.

the smallest supercell; this sampling consists in 24 different Bloch vector components in the plane of the APB and three different components along the largest side of the supercell. For the largest supercell, the irreducible wedge of the first Brillouin zone has been sampled with 35 different $k$ vectors which have the same component in the direction perpendicular to the APB.

\section{ELECTRONIC STRUCTURE NEAR THE $\{110\}(1 / 4) a_{0}[110]$ ANTIPHASE BOUNDARY}

\section{A. LSDA study of the magnetic coupling}

We have used the LSDA approximation to understand how the electronic structure depends locally on the nature of the magnetic coupling across the APB. This study has been performed with the largest supercell, which contains two APBs and two different magnetic domains which can be coupled either ferromagnetically or antiferromagnetically. The DOS near the APB which have been calculated for the two different magnetic coupling scenarios is compared to the DOS of bulk magnetite in Fig. 2. Our results confirm that bulk magnetite is a half metal, in agreement with earlier calculations using the LSDA approximation. ${ }^{18-22}$ This can be seen in the upper part of Fig. 2, which shows that the metallic behavior of bulk magnetite is only due to minority spin electrons, while the Fermi energy corresponds to the bottom of a $0.39 \mathrm{eV}$ energy gap for majority-spin electrons.

The half-metallic character is preserved in the vicinity of the APB in the case of a ferromagnetic coupling across the boundary, as shown in the central part of Fig. 2. In this case, however, the electronic structure is considerably modified in the vicinity of the structural defect. The width of the valence bands increases, and the energy gap for the majority and the minority spin are considerably smaller near the APB than in bulk magnetite. The majority-spin gaps at $-1.8 \mathrm{eV}$ below $E_{F}$ and at the Fermi energy are, for instance, respectively, reduced from 0.53 to $0.17 \mathrm{eV}$ and from 0.39 to $0.22 \mathrm{eV}$ near the APB. In the same way, the minority spin-energy gaps at $-2.96 \mathrm{eV}$ and $+1.22 \mathrm{eV}$ are, respectively, lowered from 0.18 and $0.36 \mathrm{eV}$ to 0.12 and $0.26 \mathrm{eV}$, while the small gap at $-0.57 \mathrm{eV}$ disappears at the boundary (the DOS is small but finite at this energy). The lowering of the energy gap near the APB which can be observed in Fig. 2 is due to electron states which are localized near the structural defect and cannot be coupled to the bulk states which exist on both sides of the boundary. Some of these APB states can be observed in Fig. 2 at energies inside the gap of bulk magnetite (mostly between -2.07 and $-1.53 \mathrm{eV}$, between the Fermi energy and $+0.34 \mathrm{eV}$, or above $+1.94 \mathrm{eV}$ for majority spin; between +1.04 and $1.40 \mathrm{eV}$ or above $2.56 \mathrm{eV}$ for minority spin).

Half metallicity is destroyed in the vicinity of the APB in the case of an antiferromagnetic coupling across the boundary. This can be seen in the bottom part of Fig. 2 in which all the energy gaps, including the gap at the Fermi energy, disappear at the boundary. The APB states which exist at the Fermi energy mainly involve oxygen and $\mathrm{Fe}_{B}$ atoms. The densities of majority- and minority-spin states are equal when the contributions of all the atoms are taken into account, because the supercell contains two identical magnetic domains which are coupled antiferromagnetically. The lowest part of Fig. 2 does not include all the atoms of the supercell, but only those in the APB layer and in the first atomic layer on the left-hand side of the APB. This is the reason why the majority- and minority-spin DOS are different in this figure.

The ground-state energy of the supercell which contains two APBs and two magnetic domains is found $1.35 \mathrm{eV}$ lower for the ferromagnetic than for the antiferromagnetic coupling. This energy difference only represents $6 \times 10^{-5} \%$ of the ground-state energy of the supercell. It corresponds to an energy difference of $0.675 \mathrm{eV}$ per surface $\frac{a_{0}^{2}}{\sqrt{2}}\left(1.35 \mathrm{eV} / \mathrm{nm}^{2}\right)$ for a single APB. This LSDA result indicates that ferromagnetic coupling is more stable than antiferromagnetic coupling for the $\{110\}(1 / 4) a_{0}[110]$ APB.

\section{B. LDA $+U$ calculation of the electronic structure near the $\{110\}(1 / 4) a_{0}[110]$ APB}

We have performed a LSDA $+U$ calculation of the electronic structure in the vicinity of the $\{110\}(1 / 4) a_{0}[110]$ APB. The calculation has been done with the $1.48 \mathrm{~nm}$ width supercell, assuming that the magnetic coupling across the boundary is ferromagnetic. The total DOS for a $\mathrm{Fe}_{3} \mathrm{O}_{4}$ formula unit in the vicinity of the APB is compared in Fig. 3 to the DOS calculated for bulk magnetite. The LSDA $+U$ calculation confirms the half-metallic behavior of bulk magnetite. The majority-spin energy gap calculated with the LSDA $+U$ approximation near the Fermi energy is, however, 


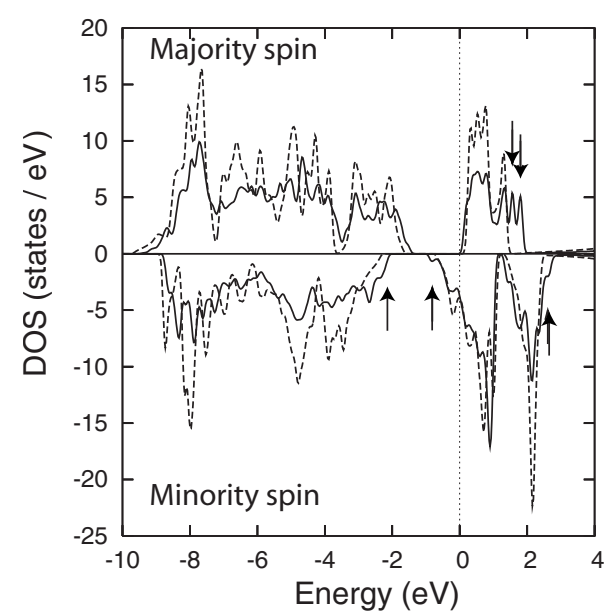

FIG. 3. Majority-spin DOS and minority-spin DOS (multiplied by -1$)$ calculated with the LSDA $+U$ approximation for bulk magnetite (dashed lines), and near a $\{110\}(1 / 4) a_{0}[110]$ APB with ferromagnetic coupling (solid lines). Vertical arrows indicate some of the DOS peaks which can be attributed to localized APB states.

larger than that obtained with the LSDA $(1.61 \mathrm{eV}$ instead of $0.39 \mathrm{eV})$. The position of the Fermi level inside this energy gap also depends on the approximation: the Fermi energy is, respectively, located at the top and at the bottom of the energy gap for the LSDA $+U$ and the LSDA calculations.

As for the LSDA calculation, the APB is responsible for the existence of localized electron states. These APB states appear as DOS peaks which do not exist in bulk magnetite. Some of these states can easily be identified because their energies are located in one of the energy gaps of bulk magnetite. This is, for instance, the case of the minority spin small peak near $-0.81 \mathrm{eV}$ and continuum of states between -2.25 and $-2.05 \mathrm{eV}$, see vertical arrows in Fig. 3. These APB states involve different kinds of atoms in the vicinity of the boundary, as shown in Fig. 4. The minority-spin DOS feature which appears just above $-2.25 \mathrm{eV}$ involves chemical bonds between $\mathrm{Fe}_{A}$ and oxygen atoms, while the peak near $-0.81 \mathrm{eV}$ is due to $\mathrm{Fe}_{B}$ and oxygen atoms near the structural defect. The majority-spin DOS peaks at 1.56 and $1.80 \mathrm{eV}$ involve $\mathrm{Fe}_{A}$ and oxygen atoms, while the minority spin peak near $2.61 \mathrm{eV}$ is built from $d$ orbitals of $\mathrm{Fe}_{B}$ atoms and $p$ orbitals of oxygen atoms. These three peaks which correspond to a linear combination of $d$ orbitals of iron and $p$ orbitals of oxygen atoms also correspond to APB states, even if they are not really located in an energy gap: they are in the same energy range as dispersive bulklike bands which give a very small contribution to the total DOS in Fig. 3; these bulklike bands are a linear combination of $s p$ orbitals of $\mathrm{Fe}_{B}$ and oxygen atoms.

The band structure of the supercell is represented in Fig. 5 for Bloch vectors along two directions of the APB plane. This figure shows the dispersion of the localized APB states along the directions [ $\overline{1} 10]$ and [001] of the magnetite grains on both sides of the boundary. The DOS peaks near $1.80 \mathrm{eV}$ for majority spin and near $2.61 \mathrm{eV}$ for minority spin, are, respectively, due to flat bands with energies between 1.5 and $1.9 \mathrm{eV}$ (for majority spin) and between 2.4 and $2.7 \mathrm{eV}$ (for minority spin). The Bloch states which are responsible for

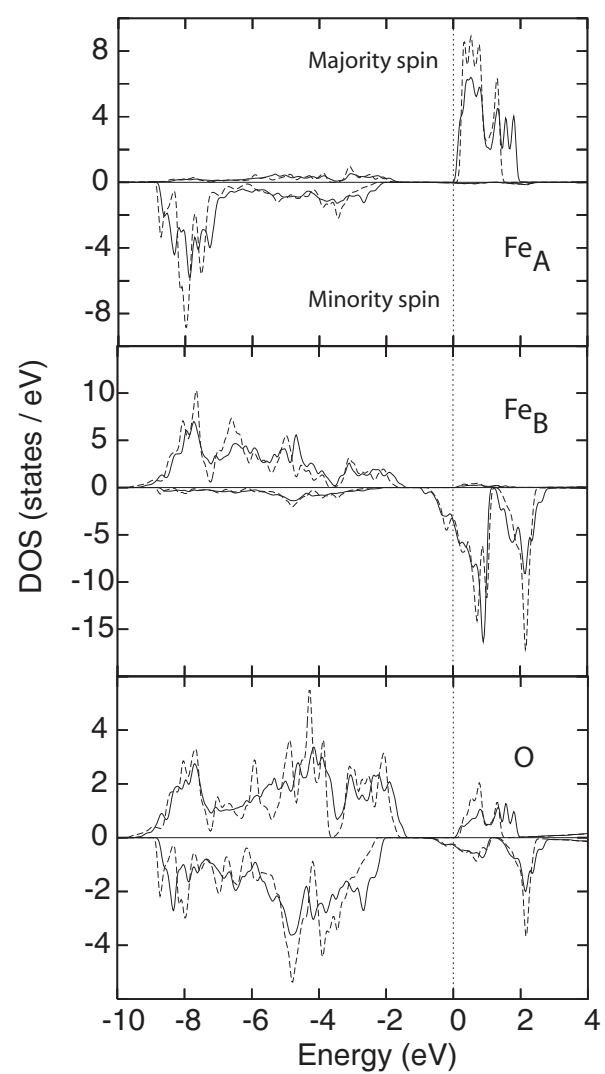

FIG. 4. $\mathrm{Fe}_{A}, \mathrm{Fe}_{B}$, and oxygen partial DOS for majority spin and minority spin (multiplied by -1 ) calculated with the LSDA $+U$ approximation for bulk magnetite (dashed lines), and near a $\{110\}(1 / 4) a_{0}[110]$ APB with ferromagnetic coupling (solid lines).

these flat bands involve oxygen and $\mathrm{Fe} d$ atomic orbitals. They cross a bulklike dispersive band (near the center of the Brillouin zone for majority-spin electrons) which is built from Fe $s p$ orbitals. The minority-spin DOS peaks just above $-2.25 \mathrm{eV}$ and near $-0.81 \mathrm{eV}$ are due to bands which show a stronger dispersion in the [110] than in the [001] direction.

We have checked that the electron states induced by APBs are strongly localized in the vicinity of these structural defects. An example of the strong localization of the APB states is shown in Fig. 6. This figure shows the electron density which corresponds to the minority-spin state with energy $-0.91 \mathrm{eV}$ at the center of the Brillouin zone $\Gamma$. The electron density is represented in two different planes which are perpendicular to the APB plane: the (001) plane which contains $\mathrm{Fe}_{B}$ atoms of the APB layer (top figure) and the (001) plane which contains $\mathrm{Fe}_{B}$ atoms first neighbors of the APB. The electron density is nearly ten times higher in this later plane, because this APB state mainly involves $d$ atomic orbitals from the $\mathrm{Fe}_{B}$ atoms in the first two atomic layers on both sides of the APB.

The spin magnetic moments are slightly modified in the vicinity of the $\mathrm{APB}$, with a small enhancement of only $0.26 \%$ and $0.98 \%$ for the $\mathrm{Fe}_{A}$ atoms and $\mathrm{Fe}_{B}$ atoms the closest from the APB. The magnetic moment of oxygen atoms is smaller than that of Fe atoms, but increases at the APB layer.

We have observed that the number of valence electrons located inside the different $\mathrm{Fe}_{B}$ atomic spheres slightly de- 


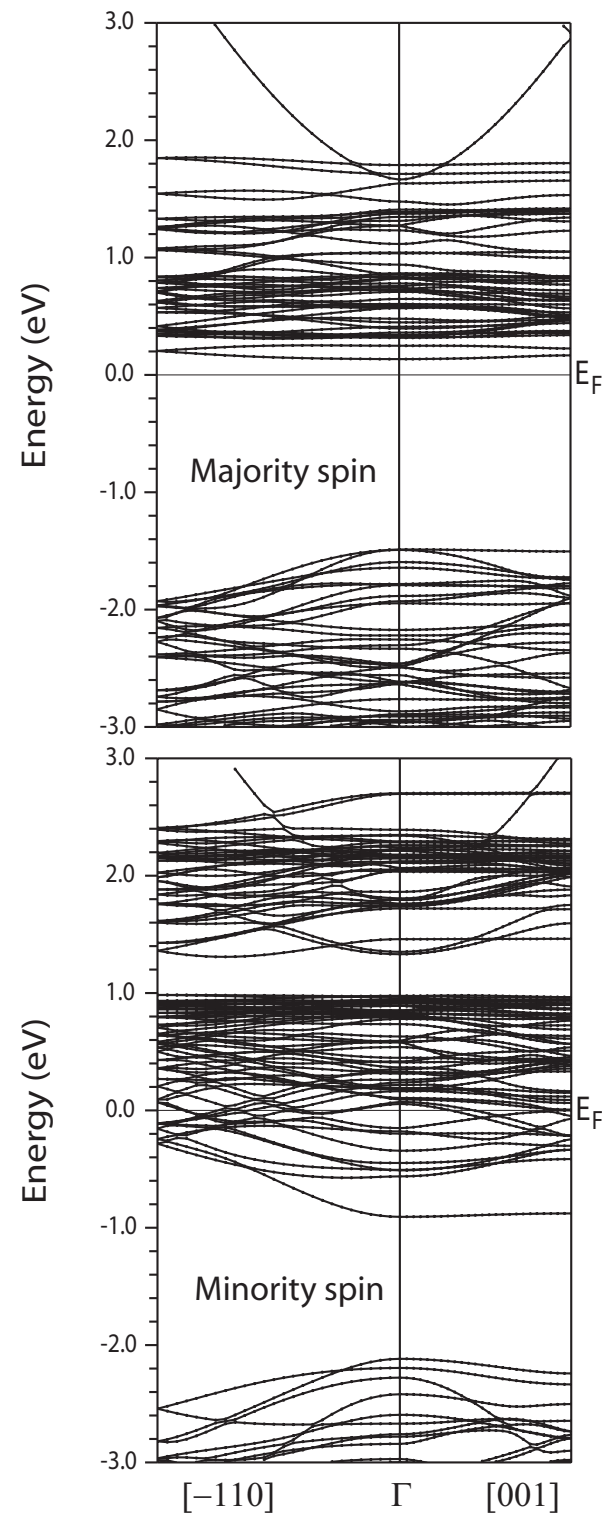

FIG. 5. Majority- and minority-spin band structure of the supercell which contains a $\{110\}(1 / 4) a_{0}[110]$ APB with ferromagnetic coupling.

pends on the distance between these atomic spheres and the APB layer. The number of majority-spin valence electrons is successively $+0.098 \%,-0.15 \%,+0.064 \%,-0.17 \%$, $+0.012 \%$, and $-0.18 \%$ higher than in bulk magnetite when the position of the $\mathrm{Fe}_{B}$ atom changes from the APB layer to the center of the magnetite slab. This small charge order along the [110] direction (the only direction in which a charge order can appear with the unit cell which has been used) is only due to the presence of the boundary. This has been checked with a bulk calculation based on a unit cell with two nonequivalent (110) atomic layers containing $\mathrm{Fe}_{B}$ atoms: the bulk ground state calculated with the $\mathrm{LDA}+U$ approximation does not show any charge order in this case. The number of minority-spin valence electrons in the $\mathrm{Fe}_{B}$ atomic spheres only changes near the APB and does not
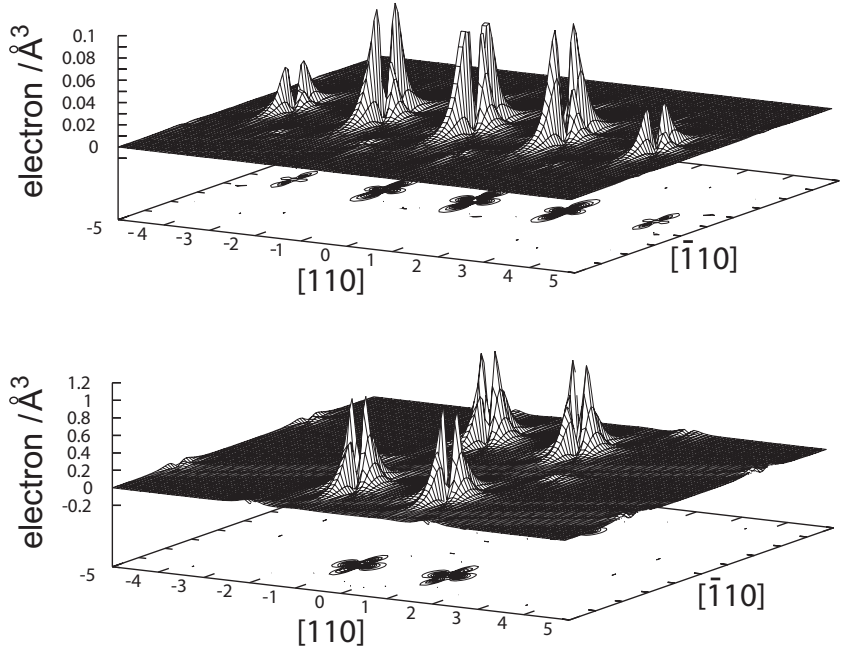

FIG. 6. Electron density for the minority-spin electron state at the center of the Brillouin zone and with an energy of $-0.91 \mathrm{eV}$ below $E_{F}$. The electron density is represented in the (001) plane which passes through $\mathrm{Fe}_{B}$ atoms of the APB layer (upper part of the figure) and in the (001) plane which passes through $\mathrm{Fe}_{B}$ atoms in the atomic layer first neighbor of the APB (lower part of the figure). The numbers along the [110] direction correspond to the successive (110) atomic layers on both sides of the APB (the APB layer being at position 0$)$.

show a significant charge order far from the APB.

\section{CONCLUSION}

We have calculated the electronic structure in the vicinity of the $\{110\}(1 / 4) a_{0}[110]$ antiphase boundary in magnetite. The LSDA approximation has first been used to compare supercells with a ferromagnetic and an antiferromagnetic coupling across this APB. Ferromagnetic coupling is found more stable than antiferromagnetic coupling with a groundstate energy difference of $1.35 \mathrm{eV} / \mathrm{nm}^{2}$ between the two situations. The calculations have also shown that half metallicity is preserved in the APB layer in the case of a ferromagnetic coupling. This point has further been confirmed by a LSDA $+U$ calculation. The density of states which has been calculated with this approximation shows that minority- and majority-spin localized electron states can exist in the vicinity of the boundaries. These APB states are built from $d$ orbitals of $\mathrm{Fe}$ atoms and $p$ orbitals of oxygen atoms and have energies with respect to the Fermi energy, near $-2.1,-0.8$, and $2.6 \mathrm{eV}$ for minority spin and near 1.6 and $1.8 \mathrm{eV}$ for majority spin. The dispersion of these states has been described in details.

\section{ACKNOWLEDGMENTS}

We acknowledge support from the ANR under Contracts No. BLAN07-188976 (SPINCHAT) and No. JCJC070138-01 (DICHROMET). The calculations presented in this article have been performed on the CALMIP/UPS Toulouse parallel computer center. 
${ }^{1}$ M. Fonin, Y. S. Dedkov, R. Pentcheva, U. Rüdiger, and G. Güntherodt, J. Phys.: Condens. Matter 19, 315217 (2007).

${ }^{2}$ E. J. Verwey and P. Haayman, Physica (Amsterdam) 8, 979 (1941).

${ }^{3}$ N. F. Mott, Philos. Mag. B 42, 327 (1980).

${ }^{4}$ A. G. Fitzgerald and R. Engin, Thin Solid Films 20, 317 (1974).

${ }^{5}$ A. G. Fitzgerald and T. G. May, Thin Solid Films 35, 201 (1976).

${ }^{6}$ W. Eerenstein, T. T. M. Palstra, T. Hibma, and S. Celotto, Phys. Rev. B 66, 201101(R) (2002).

${ }^{7}$ D. T. Margulies, F. T. Parker, M. L. Rudee, F. E. Spada, J. N. Chapman, P. R. Aitchison, and A. E. Berkowitz, Phys. Rev. Lett. 79, 5162 (1997).

${ }^{8}$ J. M. Gaines, P. J. H. Bloemen, J. T. Kohlhepp, C. W. T. BulleLieuwma, R. M. Wolf, R. M. Reinders, R. M. Jungblut, P. A. A. van der Heijden, J. T. W. M. van Eemeren, J. aan de Stegge, and W. J. M. de Jonge, Surf. Sci. 373, 85 (1997).

${ }^{9}$ S. Celotto, W. Eerenstein, and T. Hibma, Eur. Phys. J. B 36, 271 (2003).

${ }^{10}$ W. Eerenstein, Ph.D. thesis, University of Groningen, 2003.

${ }^{11}$ C. Magen, E. Snoeck, U. Lüders, and J. F. Bobo, J. Appl. Phys. 104, 013913 (2008).

${ }^{12} \mathrm{~J}$. B. Goodenough, Magnetism and the Chemical Bond (Wiley, New York, 1963).

${ }^{13}$ J. Kanamori, J. Phys. Chem. Solids 10, 87 (1959).

${ }^{14}$ P. A. A. van der Heijden, P. J. H. Bloemen, J. M. Gaines, J. T. W. M. van Eemeren, R. M. Wolf, P. J. van der Zaag, and W. J. M. de Jonge, J. Magn. Magn. Mater. 159, L293 (1996).

${ }^{15}$ D. T. Margulies, F. T. Parker, F. E. Spada, R. S. Goldman, J. Li, R. Sinclair, and A. E. Berkowitz, Phys. Rev. B 53, 9175 (1996).
${ }^{16}$ F. C. Voogt, T. T. M. Palstra, L. Niesen, O. C. Rogojanu, M. A. James, and T. Hibma, Phys. Rev. B 57, R8107 (1998).

${ }^{17}$ W. Eerenstein, T. Hibma, and S. Celotto, Phys. Rev. B 70, 184404 (2004).

${ }^{18}$ Z. Zhang and S. Satpathy, Phys. Rev. B 44, 13319 (1991).

${ }^{19}$ R. A. de Groot and K. H. J. Buschow, J. Magn. Magn. Mater. 54-57, 1377 (1986).

${ }^{20}$ M. Pénicaud, B. Siberchicot, C. B. Sommers, and J. Kübler, J. Magn. Magn. Mater. 103, 212 (1992).

${ }^{21}$ A. Yanase and N. Hamada, J. Phys. Soc. Jpn. 68, 1607 (1999).

${ }^{22}$ H.-T. Jeng and G. Y. Guo, Phys. Rev. B 65, 094429 (2002).

${ }^{23}$ Z. Szotek, W. M. Temmerman, A. Svane, L. Petit, P. Strange, G. M. Stocks, D. Ködderitzsch, W. Hergert, and H. Winter, J. Phys.: Condens. Matter 16, S5587 (2004).

${ }^{24}$ G. K. H. Madsen and P. Novak, Europhys. Lett. 69, 777 (2005).

${ }^{25}$ V. I. Anisimov, I. S. Elfimov, N. Hamada, and K. Terakura, Phys. Rev. B 54, 4387 (1996).

${ }^{26}$ P. Novák, J. Kuneš, and P. M. Oppeneer, Physica B 312-313, 785 (2002).

${ }^{27}$ D. J. Huang, C. F. Chang, H.-T. Jeng, G. Y. Guo, H.-J. Lin, W. B. Wu, H. C. Ku, A. Fujimori, Y. Takahashi, and C. T. Chen, Phys. Rev. Lett. 93, 077204 (2004).

${ }^{28}$ P. Blaha, K. Schwarz, G. K. H. Madsen, D. Kvasnicka, and J. Luitz, WIEN2k, An Augmented Plane WaveWave+Local Orbitals Program for Calculating Crystal Properties (Karlheinz Schwarz, Vienna, Austria, 2001).

${ }^{29}$ V. I. Anisimov, I. V. Solovyev, M. A. Korotin, M. T. Czyżyk, and G. A. Sawatzky, Phys. Rev. B 48, 16929 (1993).

${ }^{30}$ A. I. Liechtenstein, V. I. Anisimov, and J. Zaanen, Phys. Rev. B 52, R5467 (1995). 